\title{
Feds and industry to overhaul clinical trials
}

$\mathrm{T}$ he Canadian Institutes of Health Research (CIHR) have partnered with the pharmaceutical industry to create a national centre with the aim of overhauling Canada's clinical trials landscape. It's a bid to reverse declining research investment in the country and pare back bureaucratic barriers to starting trials.

The Canadian Clinical Trials Coordinating Centre, announced Apr. 24, will carry out An Action Plan to Help Attract More Clinical Trials to Canada, drafted with industry in 2012.

"We've lost our edge in the amount of time it takes to get a trial started here," says Jared Rhines, vice president of strategic affairs at Canada's Research-Based Pharmaceutical Companies $(R x \& D)$, a partner in the initiative. "Canada is competing for clinical research on a much larger global scale now, not only with China and India, but also with Spain, Brazil and the United Kingdom."

Canadian clinical trial activity has dropped 35\% in recent years - an estimated loss of $\$ 300$ to $\$ 500$ million per year. That decline can partly be attributed to the increasing cost and complexity of running trials in Canada, says Belinda Vandersluis, director of implementation at the new Canadian Clinical Trials Coordinating Centre.

"Clinical trial negotiations used to be quite simple and take a couple of months to complete," she explains. "That time frame has increased to the point that it now takes 6 to 18 months to negotiate a contract between a sponsor and a single site."

Companies seeking to conduct trials in Canada face a maze of disparate forms, processes, standards and contracts across jurisdictions and academic sites. Eligible patients can be difficult to recruit and retain in sufficient numbers, and research ethics reviews for the same protocols often have different requirements, results and turnaround times.

"This bureaucracy slows trials, results in trials not happening and creates disin-

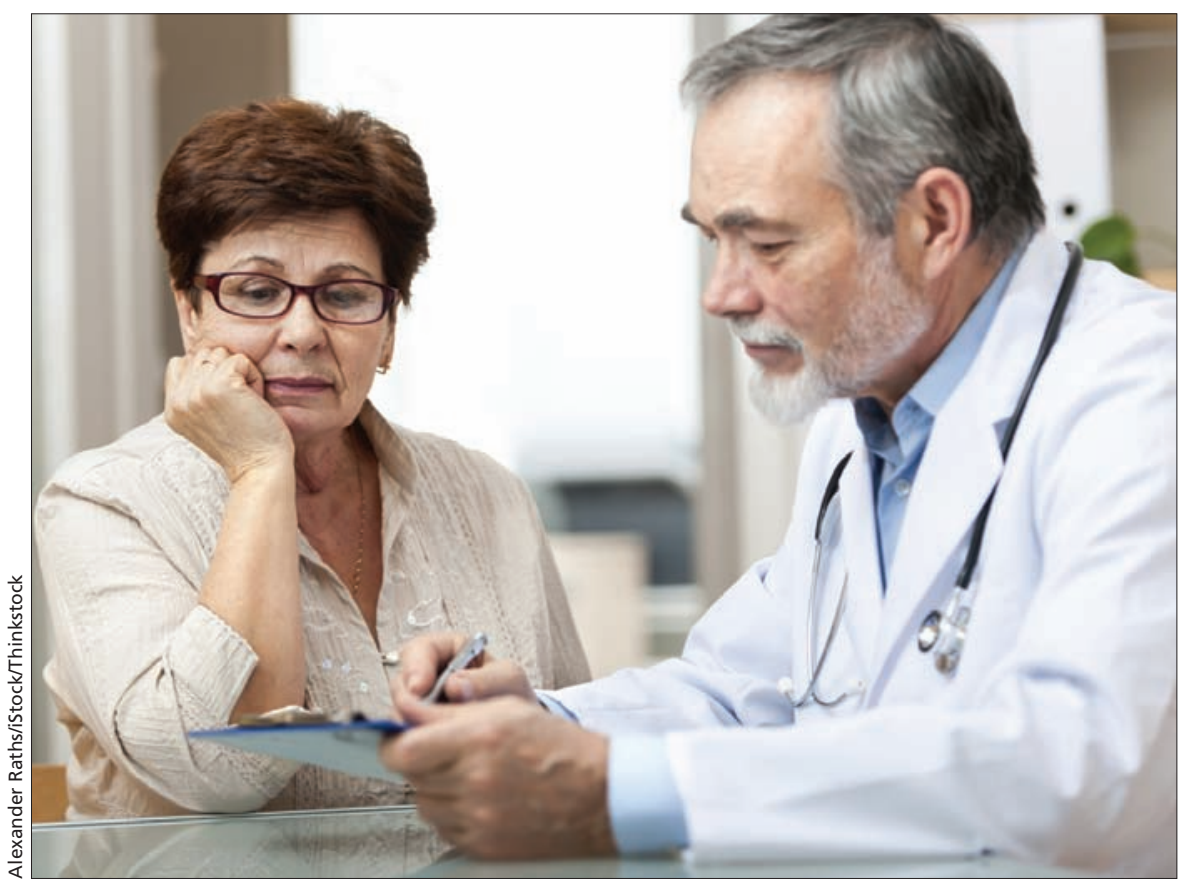

A new national centre will streamline forms, contracts and ethics review processes for clinical trials to attract more research investment to Canada.

centives for physicians to get involved in research," says Dr. P.J. Devereaux, an associate professor of clinical epidemiology and biostatistics at McMaster University in Hamilton, Ontario, who runs large-scale international trials. "We need to get to a position where we all start from the same fundamental requirements and the same basic forms so that we don't waste time and money over basically meaningless variations."

The new centre will develop common templates for contracts, consent forms, ethics review applications; metrics to monitor clinical trial performance; a national database of patient registries; standard operating practices, training and certification for academic sites; and a clinical trials "asset map" to showcase Canada's research resources to potential investors. It will also advocate for a national patient recruitment strategy and bigger incentives for drug companies, including longer patents on brand-name drugs.

Over the next three years, the federal government and Rx\&D will each pay
$\$ 750$ 000, while the merged Association of Canadian Academic Healthcare Organizations and Canadian Healthcare Association will provide $\$ 45000$ to kickstart the centre. Each partner is equally represented on the centre's governing committee, and decisions will be made by consensus.

Some health policy experts question whether this government/industry model has sufficient safeguards against undue industry influence.

"Several of these measures are long overdue, but I would have liked to see serious consideration of the ethics involved in this whole enterprise, or some acknowledgement of the potential for conflicts of interest, and I don't see attention to that," says Trudo Lemmens, Scholl Chair in Health Law and Policy at the University of Toronto.

This seems to be part of a broader shift toward closer ties with industry, he adds, citing the controversial appointments of drug company executives to CIHR's governing council in recent years. "More dependence on industry 
funding and closer collaboration with industry may make it harder for the organization to take a critical step back in the context of clinical trials."

Others note that the 2012 action plan doesn't address the potential downsides of some recommendations. For example, the benefits of stronger patent protection should be weighed against the economic and health costs of delaying the release of cheaper generic medicines, says Dr. Joel Lexchin, a health policy and management professor at York University in Toronto. Measures to address longstand- ing concerns about the concealment of clinical trial results as proprietary information are also notably missing from the plan.

However, clinical researchers contend that these concerns shouldn't preclude partnerships with industry to attract more trials to Canada.

"There should be boundaries, as there are in any relationship, but ultimately it's the responsibility of investigators to do right by their patients," says Dr. Lawrence Korngut, a neurologist at the Calgary Neuromuscular Clinic in
Alberta. His research includes largescale phase II and III clinical trials. "If you're not supported by industry, there are very few places to go to get the $\$ 20$ to $\$ 50$ million to run these trials."

"For patients with rare diseases, access to experimental treatments through clinical trials is the only hope they have," he adds. "There's a long road ahead of us and what we need to do is work together." - Lauren Vogel, CMAJ

CMAJ 2014. DOI:10.1503/cmaj.109-4811 